\title{
Correction to: Multilevel Monte Carlo front-tracking for random scalar conservation laws
}

\author{
Nils Henrik Risebro ${ }^{1}$. Christoph Schwab ${ }^{2}$. \\ Franziska Weber ${ }^{2}$
}

\section{Correction to: BIT Numer Math (2016) 56:263-292 DOI 10.1007/s10543-015-0550-4}

\begin{abstract}
An error in [4, Theorem 4.1, 4.5, Corollary 4.5] is corrected. There, in the Monte Carlo error bounds for front tracking for scalar conservation laws with random input data, 2-integrability in a Banach space of type 1 was assumed. In providing the corrected convergence rate bounds and error versus work analysis of multilevel Monte Carlo front-tracking methods, we also generalize [4] to $q$-integrability of the random entropy solution for some $1<q \leq 2$, allowing possibly infinite variance of the random entropy solutions of the scalar conservation law.
\end{abstract}

Keywords Multilevel Monte Carlo method · Conservation laws

Mathematics Subject Classification 65N30 - 65M06 · 35L65

Communicated by Lars Eldén.

The online version of the original article can be found under doi:10.1007/s10543-015-0550-4.

$凶 \quad$ Nils Henrik Risebro

nilshr@math.uio.no

Christoph Schwab

schwab@sam.math.ethz.ch

Franziska Weber

fweber@ethz.ch

1 Department of Mathematics, University of Oslo, P.O. Box 1053, 0316 Oslo, Norway

2 Seminar for Applied Mathematics, ETH Zürich, ETH Zentrum HG G 57.1, Rämistrasse 101, Zurich, Switzerland 


\section{Multilevel Monte Carlo Error Analysis}

The statement and proof of Theorem 4.1 in [4] should account for the type of Banach space in which the discretization scheme is analyzed. To render the presentation selfcontained, we recapitulate the relevant definitions as used, e.g., in [2]. For a general Banach space $E$, the type of the Banach space is defined as follows (see, e.g., [3, Page 246]).

Definition 1.1 Let $1 \leq p \leq \infty$, and $Z_{j}, j \in \mathbb{N}$, be a sequence of BernoulliRademacher random variables. A Banach space $E$ is said to be of type $p$ if there is a type constant $C>0$ such that for all finite sequences $\left(x_{j}\right)_{j=1}^{N} \subset E, N \in \mathbb{N}$,

$$
\left\|\sum_{j=1}^{N} Z_{j} x_{j}\right\|_{E} \leq C\left(\sum_{j=1}^{N}\left\|x_{j}\right\|_{E}^{p}\right)^{1 / p}
$$

Every Banach space is of type $1 ; L^{p}$-spaces are of type $\min \{p, 2\}$ for $1 \leq p<\infty$ (see [3]). The following result from [3, Proposition 9.11] for Banach spaces of type $p$ is the basis of the MLMC-FT error analysis.

Proposition 1.1 [2, Prop. 2.4] Let $E$ be a Banach space of type $p$ with type constant $C_{t}$. Then, for every finite sequence $\left(Y_{j}\right)_{j=1}^{M}$ of independent random variables in $L^{p}(\Omega ; E)$ with zero mean, one has

$$
\mathbb{E}\left[\left\|\sum_{j=1}^{N} Y_{j}\right\|_{E}^{p}\right] \leq\left(2 C_{t}\right)^{p} \sum_{j=1}^{N} \mathbb{E}\left[\left\|Y_{j}\right\|_{E}^{p}\right] .
$$

For the front-tracking error analysis, we need strengthen the assumptions from [4] to either the physical or computational domain is bounded (as, e.g., in the periodic setting) or that all realizations of the random initial data are compactly supported in $\mathbb{R}^{d}$, with support contained in one common bounded domain $D$.

\section{MLMC-FT convergence analysis}

We recapitulate notation and basic estimates from the convergence analysis from [4, Section 4.4]. In doing so, we take into account the type of the Banach spaces appearing in the error analysis which was disregarded in [4]. This results in estimates of the combined multilevel Monte Carlo front-tracking (MLMC-FT, for short) errors which differ from those in [4, Theorem 4.5, Corollary 4.5] in that more general $q$ integrability for some $1<q \leq 2$ of the random entropy solutions is considered, and that convergence rates of the front-tracking errors are now estimated in $L^{p}$ for some $p \geq q>1$. We can write 


$$
\mathbb{E}[u(t)]-E_{L}^{\mathrm{MLMC}}\left[u^{L}\right]=\mathbb{E}\left[u(t)-u^{L}(t)\right]+\mathbb{E}\left[u^{L}(t)\right]-E_{L}^{\mathrm{MLMC}}\left[u^{L}(t)\right] .
$$

Hence,

$$
\begin{aligned}
\| \mathbb{E} & {[u(t)]-E_{L}^{\mathrm{MLMC}}\left[u^{L}(t)\right] \|_{L^{q}\left(\Omega ; L^{p}\left(\mathbb{R}^{d}\right)\right)} } \\
\leq & \left\|\mathbb{E}[u(t)]-\mathbb{E}\left[u^{L}(t)\right]\right\|_{L^{q}\left(\Omega ; L^{p}\left(\mathbb{R}^{d}\right)\right)} \\
& +\left\|\mathbb{E}\left[u^{L}(t)\right]-E_{L}^{\mathrm{MLMC}}\left[u^{L}(t)\right]\right\|_{L^{q}\left(\Omega ; L^{p}\left(\mathbb{R}^{d}\right)\right)}:=A+B .
\end{aligned}
$$

We estimate the two terms $A$ and $B$. For the first one, we use the monotonicity of the expectation and the deterministic front-tracking error estimate:

$$
A=\left\|\mathbb{E}[u(t)]-\mathbb{E}\left[u^{L}(t)\right]\right\|_{L^{p}\left(\mathbb{R}^{d}\right)} \leq\left\|u(t)-u^{L}(t)\right\|_{L^{1}\left(\Omega ; L^{p}\left(\mathbb{R}^{d}\right)\right)} \leq C \delta_{L}^{s / p},
$$

where the second inequality follows from the bound on the deterministic approximation error of the FT in $L^{p}\left(\mathbb{R}^{d}\right)$. Here, $\delta_{L}$ is the FT discretization parameter at refinement level $L$. In nonadaptive approximations of the flux, $\delta_{L}=2^{-L} \delta_{0}$, where $\delta_{0}>0$ denotes the discretization parameter for the coarsest level. For front tracking, and for Lipschitz regular flux functions, $s=1$ [1]. To bound the term $B$, we set $u^{-1}:=0$ and $\Delta u^{\ell}:=u^{\ell}-u^{\ell-1}$.

$$
\begin{aligned}
& B^{q}=\left\|\mathbb{E}\left[\sum_{\ell=0}^{L}\left(u^{\ell}-u^{\ell-1}\right)\right]-\sum_{\ell=0}^{L} E_{M_{\ell}}\left(u^{\ell}-u^{\ell-1}\right)\right\|_{L^{q}\left(\Omega ; L^{p}\left(\mathbb{R}^{d}\right)\right)}^{q} \\
& =\left\|\sum_{\ell=0}^{L}\left(\mathbb{E}\left[\Delta u^{\ell}\right]-E_{M_{\ell}}\left[\Delta u^{\ell}\right]\right)\right\|_{L^{q}\left(\Omega ; L^{p}\left(\mathbb{R}^{d}\right)\right)}^{q} \\
& =\left\|\sum_{\ell=0}^{L} \sum_{j=1}^{M_{\ell}}\left(\frac{\mathbb{E}\left[\Delta u^{\ell}\right]-\Delta u^{\ell, j}}{M_{\ell}}\right)\right\|_{L^{q}\left(\Omega ; L^{p}\left(\mathbb{R}^{d}\right)\right)}^{q} \\
& \stackrel{\substack{\text { assume } \\
\stackrel{\text { Sq }}{\leq}}}{ } C_{\text {supp } u}\left\|\sum_{\ell=0}^{L} \sum_{j=1}^{M_{\ell}}\left(\frac{\mathbb{E}\left[\Delta u^{\ell}\right]-\Delta u^{\ell, j}}{M_{\ell}}\right)\right\|_{L^{q}\left(\Omega ; L^{q}\left(\mathbb{R}^{d}\right)\right)}^{q} .
\end{aligned}
$$

Define

$$
Y^{\ell, j}:=\frac{\mathbb{E}\left[\Delta u^{\ell}\right]-\Delta u^{\ell, j}}{M_{\ell}} .
$$

$Y^{\ell, j}$ are independent, zero-mean random variables. Hence, we can use [3, Prop. 9.11] as stated in Proposition 1.1 (see also [2, Cor. 2.5]) for $q \in(1,2]$ to estimate $B^{q}$ by: 


$$
\begin{gathered}
B^{q} \leq C_{\operatorname{supp} u} C_{q} \sum_{\ell=0}^{L} \sum_{j=1}^{M_{\ell}}\left\|Y^{\ell, j}\right\|_{L^{q}\left(\Omega ; L^{q}\left(\mathbb{R}^{d}\right)\right)}^{q} \\
:=\sum_{\ell=0}^{L} \sum_{j=1}^{M_{\ell}}\left\|\frac{\mathbb{E}\left[\Delta u^{\ell}\right]-\Delta u^{\ell, j}}{M_{\ell}}\right\|_{L^{q}\left(\Omega ; L^{q}\left(\mathbb{R}^{d}\right)\right)}^{q},
\end{gathered}
$$

where $C_{q}$ is a constant depending on the type $q$ of the Banach space $L^{q}$. We continue estimating the last term:

$$
\begin{aligned}
& B^{q} \leq C_{\text {supp } u} C_{q} \sum_{\ell=0}^{L} M_{\ell}^{1-q}\left\|\mathbb{E}\left[\Delta u^{\ell}\right]-\Delta u^{\ell, 1}\right\|_{L^{q}\left(\Omega ; L^{q}\left(\mathbb{R}^{d}\right)\right)}^{q} \\
& \leq C_{\text {supp } u} C_{q} \sum_{\ell=0}^{L} M_{\ell}^{1-q}\left\|\Delta u^{\ell, 1}\right\|_{L^{q}\left(\Omega ; L^{q}\left(\mathbb{R}^{d}\right)\right)}^{q} \\
& \leq C_{\text {supp } u, q}\left(M_{0}^{1-q}\right. \\
& \left.+\sum_{\ell=1}^{L}\left(\frac{\left\|u^{\ell}(t)-u(t)\right\|_{L^{q}\left(\Omega ; L^{q}\left(\mathbb{R}^{d}\right)\right)}^{q}}{M_{\ell}^{1-q}}+\frac{\left\|u^{\ell-1}(t)-u(t)\right\|_{L^{q}\left(\Omega ; L^{q}\left(\mathbb{R}^{d}\right)\right)}^{q}}{M_{\ell}^{1-q}}\right)\right) \\
& \leq C_{\text {supp }, q}\left(M_{0}^{1-q}+\sum_{\ell=1}^{L} M_{\ell}^{1-q}\left\|u^{\ell}(t)-u(t)\right\|_{L^{q}\left(\Omega ; L^{q}\left(\mathbb{R}^{d}\right)\right)}^{q}\right) \\
& \stackrel{\text { Hölder }}{\leq} C_{\text {supp } u, q}\left(M_{0}^{1-q}+C_{\left\|u_{0}\right\|_{\infty}} \sum_{\ell=1}^{L} M_{\ell}^{1-q}\left\|u^{\ell}(t)-u(t)\right\|_{L^{q}\left(\Omega ; L^{1}\left(\mathbb{R}^{d}\right)\right)}\right) \\
& \text { deterministic } \\
& \stackrel{\substack{\text { error } \\
\text { estimate }}}{\leq} C\left(\operatorname{supp} u, q,\left\|u_{0}\right\|_{\infty}, T,\|f\|_{W^{1, \infty}}\right)\left(M_{0}^{1-q}+\sum_{\ell=1}^{L} M_{\ell}^{1-q} \delta_{\ell}^{s}\right) \text {, }
\end{aligned}
$$

where we recall that $\delta_{\ell}$ is the FT flux discretization parameter at level $\ell, \ell=0, \ldots, L$, and that for front tracking, the convergence rate is $s=1$.

Combining the estimates for terms $A$ and $B$, we arrive at

$$
\left\|\mathbb{E}[u]-E_{L}^{\mathrm{MLMC}}\left[u^{L}\right]\right\|_{L^{q}\left(\Omega ; L^{p}\left(\mathbb{R}^{d}\right)\right)}^{q} \leq C\left(M_{0}^{1-q}+\sum_{\ell=1}^{L} M_{\ell}^{1-q} \delta_{\ell}^{S}+\delta_{L}^{\frac{s q}{p}}\right) .
$$

For $p=1$, the last term is minimized which results in the bound

$$
\left\|\mathbb{E}[u]-E_{L}^{\mathrm{MLMC}}\left[u^{L}\right]\right\|_{L^{q}\left(\Omega ; L^{1}\left(\mathbb{R}^{d}\right)\right)}^{q} \leq C\left(M_{0}^{1-q}+\sum_{\ell=1}^{L} M_{\ell}^{1-q} \delta_{\ell}^{s}+\delta_{L}^{s q}\right) .
$$




\section{Choice of MC sample sizes $M_{\ell}$ and error versus work bounds}

The Monte Carlo sample numbers in the error bound (2.2), i.e., $M_{\ell}$, at FT discretization level $\ell$ should be chosen such that for a given error tolerance $\varepsilon$, the bound for the required computational work is optimized. We recall, from [4], the work estimates for the deterministic front-tracking method for different scenarios:

Proposition 3.1 The computational work $W_{F T}$ for the deterministic front-tracking method behaves asymptotically as

$$
W_{F T} \simeq \delta^{-w},
$$

where $\delta>0$ is the discretization parameter and $w$ given by

$$
w= \begin{cases}1, & d=1, \text { convex flux }, \\ 2, & d=1, \text { nonconvex flux } \\ 2 d+1, & d>1, \text { convex flux components } \\ 2 d+2, & d>1, \text { nonconvex flux components }\end{cases}
$$

The computational work for the multilevel Monte Carlo front-tracking scheme behaves asymptotically as

$$
W_{L}^{M L M C-F T} \simeq \sum_{\ell=0}^{L} M_{\ell} \delta_{\ell}^{-w},
$$

with the work rate $w$ as given in (3.1).

For a given maximal discretization level $L$ and for prescribed overall error $\varepsilon>0$, the choice of sample numbers $M_{\ell}$ is obtained by optimizing the error bounds, as is by now customary in MLMC error analysis. The following result is analogous to Lemma 4.9 in [4]:

Lemma 3.1 Let $\left\{\delta_{\ell}\right\}_{\ell \geq 0}$ be a strictly decreasing sequence of positive FT discretization parameters. Assume that the work for the MLMC-FT algorithm with L discretization levels scales asymptotically as in (3.2), that is, there exists a constant $C>0$ which is independent of $\left\{\delta_{\ell}\right\}_{\ell \geq 0}$ and of $\left\{M_{\ell}\right\}_{\ell \geq 0}$ such that for every $L \geq 1$,

$$
W_{L}^{M L M C} \leq C \sum_{\ell=0}^{L} M_{\ell} \delta_{\ell}^{-w},
$$

for $w>0$, given in (3.1). Given that the MCFT error at FT discretization level L is bounded as [cf. (2.2)]

$$
\operatorname{Err}_{L} \leq C\left(\sum_{\ell=0}^{L} M_{\ell}^{1-q} \delta_{\ell}^{q s}+\delta_{L}^{q s}+M_{0}^{1-q}\right)
$$


(i.e., $p=1$ ), the optimal sample numbers $M_{\ell}$ in terms of the work bound (3.3) and of the error bound (3.4) are given by

$$
M_{\ell} \simeq M_{0} \delta_{0}^{-\frac{w}{q}} \delta_{\ell}^{\frac{s+w}{q}}, \quad \ell=1, \ldots, L
$$

where

$$
M_{0} \simeq\left(\frac{\delta_{0}^{\frac{w-w q}{q}}+\sum_{j=1}^{L} \delta_{j}^{\frac{s+w-w q}{q}}}{\varepsilon-\delta_{L}^{q s}}\right)^{\frac{1}{q-1}} \delta_{0}^{\frac{w}{q}} .
$$

The symbol $\simeq$ indicates equality up to a constant which may depend on the data $\left(u_{0}, f\right)$ and the domain but which is independent of $\ell$ and $L$. If we assume in addition that the $\delta_{\ell}$ are a geometric sequence, that is, $\delta_{\ell}=2^{-\alpha \ell} \delta_{0}$ for some $\alpha, \delta_{0}>0$, we obtain for $L \rightarrow \infty$ that the error of the MLMC algorithm is bounded for any $s>0$, and for $q \in(1,2]$ as

$$
\begin{aligned}
& \| \mathbb{E}[u(\cdot, t)]-E_{L}^{M L M C}[u(\cdot, t)] \|_{L^{q}\left(\Omega ; L^{1}\left(\mathbb{R}^{d}\right)\right)} \\
& \leq C \begin{cases}\left(W_{L}^{M L M C}\right)^{-\frac{s}{s+w}}, & s+w-w q<0, \\
\left(W_{L}^{M L M C}\right)^{-\frac{q-1}{q}} \log \left(W_{L}^{M L M C}\right), & s+w-w q=0, \\
\left(W_{L}^{M L M C} W_{0, \mathrm{det}}\right)^{-\frac{q-1}{q}}, & s+w-w q>0,\end{cases}
\end{aligned}
$$

where $W_{0, \mathrm{det}}$ is the work for the deterministic front-tracking algorithm with resolution $\delta_{0}>0$. In particular, the error of the MLMC-FT algorithm $(s=1)$ scales for $p=1$, $q=2$ with respect to work as

$$
\begin{aligned}
&\left\|\mathbb{E}[u(\cdot, t)]-E_{L}^{M L M C}[u(\cdot, t)]\right\|_{L^{2}\left(\Omega ; L^{1}\left(\mathbb{R}^{d}\right)\right)} \\
& \simeq \begin{cases}\left(W_{L}^{M L M C}\right)^{-1 / 2} \log \left(W_{L}^{M L M C}\right), & d=1, \text { convex flux } \\
\left(W_{L}^{M L M C}\right)^{-1 / 3}, & d=1, \text { nonconvex flux }, \\
\left(W_{L}^{M L M C}\right)^{-\frac{1}{2+2 d}}, & d>1 \text { convex flux components }, \\
\left(W_{L}^{M L M C}\right)^{-\frac{1}{3+2 d}}, & d>1 \text { nonconvex flux components. }\end{cases}
\end{aligned}
$$

Proof We use a Lagrange multiplier argument to determine the optimal $M_{\ell}$. Since the assertion is about an asymptotically optimal error versus work bound, we require an (eventually small) error tolerance $\varepsilon>0$ and assume all constants in the error bounds to take the value 1 . This assumption results in the following asymptotic work and error measures:

$$
\begin{aligned}
W_{L} & :=\sum_{\ell=0}^{L} M_{\ell} \delta_{\ell}^{-w} \\
\operatorname{Err}_{L} & :=M_{0}^{1-q}+\delta_{L}^{q s}+\sum_{\ell=1}^{L} M_{\ell}^{1-q} \delta_{\ell}^{s}
\end{aligned}
$$


To minimize the overall work subject to prescribed error $\varepsilon>0$, consider the Lagrangian

$$
\mathscr{L}:=W_{L}-\lambda\left(\varepsilon-\operatorname{Err}_{L}\right)
$$

where $\lambda$ is the Lagrange multiplier. Taking the derivative of $\mathscr{L}$ with respect to $M_{\ell}$, we have

$$
\begin{aligned}
\frac{\partial \mathscr{L}}{\partial M_{\ell}} & =\frac{\partial W_{L}}{\partial M_{\ell}}+\lambda \frac{\partial \operatorname{Err}_{L}}{\partial M_{\ell}}, \\
\frac{\partial W_{L}}{\partial M_{\ell}} & =\delta_{\ell}^{-w}, \\
\frac{\partial \operatorname{Err}_{L}}{\partial M_{\ell}} & = \begin{cases}(1-q) M_{\ell}^{-q} \delta_{\ell}^{s}, & \ell>0, \\
(1-q) M_{0}^{-q}, & \ell=0 .\end{cases}
\end{aligned}
$$

Hence,

$$
\delta_{\ell}^{-w}= \begin{cases}\lambda(q-1) M_{\ell}^{-q} \delta_{\ell}^{s}, & \ell>0 \\ \lambda(q-1) M_{0}^{-q}, & \ell=0\end{cases}
$$

or

$$
M_{\ell}= \begin{cases}\left(\delta_{\ell}^{s+w} \lambda(q-1)\right)^{\frac{1}{q}}, & \ell>0, \\ \left(\delta_{0}^{w} \lambda(q-1)\right)^{\frac{1}{q}}, & \ell=0 .\end{cases}
$$

Now insert $\varepsilon=\operatorname{Err}_{L}$ to get an expression for $\lambda$ :

$$
\begin{aligned}
\varepsilon=\operatorname{Err}_{L} & =\delta_{L}^{q s}+\left(\delta_{0}^{w} \lambda(q-1)\right)^{\frac{1-q}{q}}+(\lambda(q-1))^{\frac{1-q}{q}}\left(\sum_{\ell=1}^{L} \delta_{\ell}^{\frac{s+w}{q}(1-q)} \delta_{\ell}^{s}\right) \\
& =\delta_{L}^{q s}+(\lambda(q-1))^{\frac{1-q}{q}}\left(\delta_{0}^{\frac{w(1-q)}{q}}+\sum_{\ell=1}^{L} \delta_{\ell}^{\frac{s+w-w q}{q}}\right) .
\end{aligned}
$$

We solve this for $\lambda$ :

$$
\lambda=\frac{1}{q-1}\left(\frac{\delta_{0}^{\frac{w-w q}{q}}+\sum_{\ell=1}^{L} \delta_{\ell}^{\frac{s+w-w q}{q}}}{\varepsilon-\delta_{L}^{q s}}\right)^{\frac{q}{q-1}} .
$$

The sample numbers $M_{\ell}$ are given by

$$
M_{\ell}= \begin{cases}\left(\frac{\delta_{0}^{\frac{w-w q}{q}}+\sum_{j=1}^{L} \delta_{j}^{\frac{s+w-w q}{q}}}{\varepsilon-\delta_{L}^{q s}}\right)^{\frac{1}{q-1}} \delta_{\ell}^{\frac{s+w}{q}}, \quad \ell>0, \\ \left(\frac{\delta_{0}^{\frac{w-w q}{q}}+\sum_{j=1}^{L} \delta_{j}^{\frac{s+w-w q}{q}}}{\varepsilon-\delta_{L}^{q s}}\right)^{\frac{1}{q-1}} \delta_{0}^{\frac{w}{q}}, \quad \ell=0,\end{cases}
$$


and the bound for the overall work becomes

$$
\begin{aligned}
W_{L} & :=\sum_{\ell=0}^{L} M_{\ell} \delta_{\ell}^{-w} \\
& =\left(\frac{\delta_{0}^{\frac{w-w q}{q}}+\sum_{j=1}^{L} \delta_{j}^{\frac{s+w-w q}{q}}}{\varepsilon-\delta_{L}^{q s}}\right)^{\frac{1}{q-1}}\left(\delta_{0}^{\frac{w-w q}{q}}+\sum_{\ell=1}^{L} \delta_{\ell}^{\frac{s+w-w q}{q}}\right) \\
& =\left(\frac{1}{\varepsilon-\delta_{L}^{q s}}\right)^{\frac{1}{q-1}}\left(\delta_{0}^{\frac{w-w q}{q}}+\sum_{\ell=1}^{L} \delta_{\ell}^{\frac{s+w-w q}{q}}\right)^{\frac{q}{q-1}} .
\end{aligned}
$$

Now choose $\varepsilon \simeq \delta_{L}^{q s}$, i.e., $\varepsilon=2 \delta_{L}^{q s}$, to balance the two error contributions and obtain

$$
W_{L}=\delta_{L}^{-\frac{q s}{q-1}}\left(\delta_{0}^{\frac{w-w q}{q}}+\sum_{\ell=1}^{L} \delta_{\ell}^{\frac{s+w-w q}{q}}\right)^{\frac{q}{q-1}}
$$

Now let us assume again that $\delta_{\ell} \simeq 2^{-\alpha \ell}$ with some $\alpha>0$, i.e., the $\delta_{\ell}$ are geometrically decreasing to zero. We distinguish three cases:

1. Case $s+w-w q<0\left(\Leftrightarrow q>\frac{s}{w}+1\right)$ : Then

$$
W_{L} \simeq \delta_{L}^{-\frac{q s}{q-1}} \delta_{L}^{\frac{s+w-w q}{q-1}}=\delta_{L}^{-(s+w)}
$$

hence

$$
\left(\operatorname{Err}_{L}\right)^{1 / q} \simeq \delta_{L}^{s} \simeq W_{L}^{-\frac{s}{s+w}} .
$$

2. Case $s+w-w q=0\left(\Leftrightarrow q=\frac{s}{w}+1\right)$ : Then

$$
W_{L} \simeq \delta_{L}^{-\frac{q s}{q-1}} L^{\frac{q}{q-1}}
$$

hence

$$
\begin{gathered}
W_{L}\left(\log W_{L}\right)^{-\frac{q}{q-1}} \simeq \delta_{L}^{-\frac{q s}{q-1}} \\
\left(\operatorname{Err}_{L}\right)^{1 / q} \simeq \delta_{L}^{S} \simeq\left(W_{L}\left(\log W_{L}\right)^{-\frac{q}{q-1}}\right)^{-\frac{q-1}{q}} \simeq W_{L}^{-\frac{q-1}{q}} \log W_{L} .
\end{gathered}
$$

3. Case $s+w-w q>0\left(\Leftrightarrow q<\frac{s}{w}+1\right)$ : Then

$$
W_{L} \simeq \delta_{L}^{-\frac{q s}{q-1}}\left(\delta_{0}^{\frac{w(1-q)}{q}}\right)^{\frac{q}{q-1}}=\delta_{L}^{-\frac{q s}{q-1}} \delta_{0}^{-w}
$$


Note that $\delta_{0}^{-w} \simeq W_{0, \text { det. }}$ Hence,

$$
\left(\operatorname{Err}_{L}\right)^{1 / q} \simeq \delta_{L}^{s} \simeq\left(W_{L} W_{0, \mathrm{det}}^{-1}\right)^{-\frac{q-1}{q}}
$$

For the MLMC front-tracking method, this implies according to Proposition 3.1 for $q=2$,

$$
\operatorname{Err}_{L}^{1 / q} \simeq \begin{cases}W_{L}^{-1 / 2} \log W_{L}, & d=1, \text { convex flux } \\ W_{L}^{-1 / 3}, & d=1, \text { nonconvex flux } \\ W_{L}^{-\frac{1}{2+2 d}}, & d>1, \text { convex flux components } \\ W_{L}^{-\frac{1}{3+2 d}}, & d>1, \text { nonconvex flux components. }\end{cases}
$$

\section{References}

1. Holden, H., Risebro, N.H.: Front tracking for hyperbolic conservation laws. Applied Mathematical Sciences, vol. 152. Springer, New York (2011). First softcover corrected printing of the 2002 original

2. Koley, U., Risebro, N.H., Schwab, C., Weber, F.: A multilevel Monte Carlo finite difference method for random scalar degenerate convection diffusion equations. J. Hyper. Diff. Eqns (to appear)

3. Ledoux, M., Talagrand, M.: Probability in Banach spaces. Classics in Mathematics. Springer, Berlin (2011). Isoperimetry and processes, Reprint of the 1991 edition

4. Risebro, N.H., Schwab, C., Weber, F.: Multilevel Monte Carlo front-tracking for random scalar conservation laws. BIT Numer. Math. 1-30 (2015) 